\title{
Intervención en las problemáticas familiares, barrio La Divisa, Comuna 13 - Medellín ${ }^{1}$
}

\author{
Intervention in family issues, la divisa neighborhood, comuna 13 - Medellín \\ Intervenção nas problemáticas familiares no bairro la divisa, cidade de Medellín
}

DOI: https://doi.org/10.21803/pensam.v11i21-1.259

Jenniffer Ximena Vega Fajardo http://orcid.org/0000- 0001-6733-891X

Sonia Lucía Vargas Amézquita http://orcid.org/0000-0003-4813

\section{¿Cómo citar este artículo?}

Vega, J. \& Vargas, S. (2018). Intervención en las problemáticas familiares, barrio La Divisa, Comuna 13 - Medellín. Pensamiento Americano, $11(22), 17-24$

17

https://doi.org/10.21803/pensam.v17i21-1.259

\begin{abstract}
Resumen
Este estudio presenta la intervención en los aspectos emocionales, socioculturales y cognitivos de las diferentes problemáticas encontradas en las familias del Barrio la Divisa de la ciudad de Medellín bajo el enfoque de investigación cualitativa, con diseño investigación, acción participativa, dividida en tres fases: diagnóstico, planeación - ejecución, y evaluación.
\end{abstract}

Se lograron diseñar estrategias para atender cada una de las necesidades que se percibieron en la comunidad en la fase diagnóstica desde siete microproyectos y se realizó la ejecución de estos, llevando actividades semanales a 45 niños y sus familias quienes participaron libremente del proyecto. Para la tercera fase se espera realizar la evaluación de impacto social de la intervención realizada.

Palabras Clave: Intervención social, familia, infancia.

\begin{abstract}
This study presents the intervention in the emotional, sociocultural and cognitive aspects of the different problems found in the families of the Barrio la Divisa from Medellín. This paper has a qualitative focus; the research design is action - participatory, divided into three phases: diagnosis, planning - execution, and evaluation.

Strategies were designed to address the needs that were perceived in the community in the diagnostic phase from seven micro-projects. The execution of such micro-projects was carried out, taking weekly activities to 45 children and their families who participated freely in the project. For the third phase, it is expected to carry out the social impact evaluation of the performed intervention.
\end{abstract}

Keywords: Social intervention, family, childhood.

\footnotetext{
1. Intervención en las Problemáticas Familiares en la Comuna 13 Barrio La Divisa en la Ciudad de Medellín, Entidades financiadoras: Corporación Universitaria Adventista, Asociación Centro Occidental de los Adventistas del Séptimo Día. Inicio del Proyecto: Fase Uno: Febrero de 2016 - Noviembre de 2016, Fase Dos: Febrero 2017 - Noviembre 2017, Fase Tres: Febrero 2018 - Noviembre 2018.
} 


\section{Resumo}

Este estudo apresenta a intervenção nos aspectos emocionais, socioculturais e cognitivos das diferentes problemáticas encontradas nas famílias do Bairro La Divisa da cidade de Medellín sob o enfoque de investigação qualitativa, com desenho, investigação, ação participativa, dividida em três fases: diagnóstico, planejamento -execução e avaliação. Conseguiu-se desenhar estratégias para atender cada uma das necessidades que foram percebidas na comunidade na fase de diagnóstico desde sete microprojetos e sua posterior realização, efetuando atividades semanais a 45 crianças e suas famílias que participaram livremente do projeto. Para a terceira fase, se espera realizar a avaliação do impacto social da intervenção realizada.

Palavras chave: Intervenção Social; Família; Infância.

\section{Perfil}

Licenciada en Preescolar. Especialista en Intervenciones Psicosociales. Magíster en Educación con mención en Psicología Educativa. Asesora de investigación. Corporación Universitaria Adventista.

jvega@unac.edu.co

\section{Perfil}

Coordinadora Licenciatura en Preescolar. Docente Facultad de Educación. Especialista en Pedagogía e Investigación en el Aula. Investigadora del grupo Pedagogía Cultura y Sociedad de la Facultad de Educación. Líder de la Línea Pedagogía e Infancia de la Corporación Universitaria Adventista.

svargas@unac.edu.co.

\section{Jenniffer Ximena} Vega Fajardo

Magíster en Educación con mención en Psicología Educativa.

\section{Sonia Lucía} Vargas Amézquita

Mg. En Neuropsicologia Educativa. 


\section{Introducción}

E plan de desarrollo local de la comuna 13, describe las características que han marcado a la comunidad. Sus habitantes, durante los últimos 30 años han sido atravesados y confrontados con los rigores del conflicto armado, los fenómenos de desplazamiento intraurbano, la pobreza generalizada, las pocas condiciones ambientales, la violación de los derechos, todo lo cual ha generado inestabilidad social y un ambiente de incertidumbre colectiva, que sitúa a un gran número de sus habitantes en condiciones de vulnerabilidad y en un estado de subsistencia indigno de la condición humana (Alcaldía de Medellín, 2010).

Los factores mencionados anteriormente por el Plan de Desarrollo Local de la Comuna 13 , son las causas principales para que se genere un gran índice de analfabetismo, desempleo, pobreza, déficit de vivienda, desnutrición infantil, etc. Lo que a su vez aumenta el número de conflictos que se afrontan en la comuna y la familia.

La comuna 13 busca que los ciudadanos participen de una forma activa en los cambios del tejido social y que dichos cambios sean reconocidos a nivel local, nacional e internacional. El Plan de Desarrollo Local Comuna 13 pretende poner en marcha diversos proyectos y programas que ayuden a resolver las problemáticas sociales y que a su vez contribuyan al cambio de la concepción que tiene el resto del país frente a la comuna 13 , que ésta pase de ser un emblema de violencia a una insignia de paz donde se vea proyectado un desarrollo integral y una calidad de vida mejorada.

Dada la problemática anterior, la Licenciatura en Preescolar de la Corporación Universitaria
Adventista, dentro del alcance de sus objetivos al buscar mecanismos para la identificación de dificultades a nivel cognitivo, social, emocional o espiritual, para aportar solucionesy estrategias de intervención desde la docencia, la investigación y la proyección social a la primera infancia y padres de familia, propone la presente investigación, con el fin de mejorar la calidad de vida centrándose en el núcleo principal de la sociedad: la familia; y haciendo a cada individuo participe en la transformación de la realidad familiar que llevará al cambio del tejido social. Dichos esfuerzos integrarán la participación de profesionales de la Corporación Universitaria Adventista y de los habitantes de la comuna 13, cumpliendo así las visiones de estos dos entes.

Las principales necesidades encontradas se agrupan en tres grandes áreas: en el desarrollo cognitivo, en el desarrollo afectivo y en el desarrollo sociocultural.

\section{Objetivo general y objetivos específicos}

- Intervenir en las problemáticas familiares de la comuna 13, barrio La Divisa en la ciudad de Medellín que afectan el desarrollo de las familias.

- Identificar conductas que muestren las necesidades cognitivas, afectivas y socioculturales de los niños pertenecientes a familias del barrio La Divisa en la ciudad de Medellín.

- Diseñar estrategias que permitan atender las necesidades en los aspectos socioculturales, afectivos y cognitivos de las familias de la comuna 13, barrio La Divisa en la ciudad de Medellín.

- Fortalecer a las familias de la comuna 13, barrio La Divisa en la ciudad de Medellín, por medio de las cartillas y las actividades realizadas en el CIF?. 


\section{Marco teórico}

\section{La familia}

Reconociendo a la familia como núcleo fundamental de la sociedad, y el papel juega dentro de ella se hace necesario considerar algunos conceptos o definiciones sobre la familia. Enel2013, LaAlcaldía deMedellín, como se citó en García (2013), asume el concepto de familia como un subsistema social abierto que hace parte de un suprasistema denominado sociedad, al interior del cual se establecen y configuran relaciones dialécticas entre la familia y con los otros subsistemas sociales, lo que origina en estos cambios y trasformación de orden configurativo, estructural, dinámico y funcional (p. 53).

Dicho de otra manera, la familia hace parte de la sociedad, y es allí, donde hay intercambios de razonamientos y argumentaciones entre sí y con otros subsistemas. Además, la familia es un sistema vivo de la sociedad que está en constante cambios. Con respecto a esto, Belart y Ferrer (2012) manifiesta que:

La familia es un sistema, es decir, un grupo cuyos miembros están interrelacionados. $Y$ es un sistema vivo y dinámico en constante transformación: vida y cambio van inseparablemente unidos. La característica principal de cualquier sistema vivo es la tendencia al crecimiento: nacer, crecer, reproducirse y morir. Por tanto, la familia, como sistema vivo, está constantemente sometida a cambios. Seamos conscientes de que todo cambia y nada permanece, la vida es como un río que fluye sin cesar (p.26).

Dentro de esta investigación se ha decidido destacar tres aspectos fundamentales para el desarrollo del individuo (social, cognitivo y emocional o afectivo), los cuales están respaldados por diferentes teóricos. En primera instancia se hablará del desarrollo social.

\section{Desarrollo social}

A través del tiempo muchos autores han definido el desarrollo humano desde diferentes puntos de vista, en esta ocasión se tomará como referente la teoría de la ecología del desarrollo humano expuesta por Urie Bronfenbrenner (1987). Según su teoría cada persona es afectada de modo significativo por las interacciones de una serie de sistemas que se superponen; esta teoría inicia definiendo el desarrollo humano como:

El proceso por el cual la persona en desarrollo adquiere una concepción de ambiente ecológico más amplia, diferenciada y valida, y se motiva, vuelve capaz de realizar actividades que revelen las propiedades del ambiente, lo apoyen y lo reestructuren, a niveles de igual y mayor complejidad, en cuanto a su forma y contenido (Bronfenbrenner, 1987, p. 47).

De lo anterior, se entiende que el desarrollo es un proceso en el cual el ser humano se ve vinculado con el ambiente, proveyendo la interacción, y al mismo tiempo, originando acciones de participación con el medio. Es por ello que esta teoría forma parte de las teorías dialécticas contextuales, las cuales explican el cambio de conducta del individuo a través de la influencia del entorno o medio, convirtiéndose así en un cambio multidireccional, multicontextual y multicultural.

\section{Desarrollo afectivo}

Es claro que uno de los componentes más importantes en el desarrollo afectivo es el concepto de apego, ya que éste juega un papel fundamental en la parte afectiva de todo ser humano. Por esto, es crucial definir y dialogar sobre este tema, para lo que se 
utilizarán los aportes de diferentes autores que hablan sobre la obra de John Bowlby (2014). Para comenzar resulta adecuado citar la definición del apego, como:

Una "reacción, conducta instintiva y trascendental para el primer año de vida". Significa también una relación de "reciprocidad" entre la diada, como un aprendizaje para la conducta de relación futura. La figura de apego, es la madre o figura sustituta, padre, tía, abuela, entre otras. Sostenía que "el apego acorta la distancia afectiva y lo podemos apreciar a través de la succión, aferramiento, seguimiento, llanto, sonrisa". Y afirmaba que el apego es más importante para sobrevivir que la comida y la reproducción. (Bowlby citado por Maglio y Graiño, 2015, párr. 2).

En efecto, se puede decir que la figura de apego debe ser estable y debe suplir las necesidades emocionales del niño, además de resaltar la importancia que esta presenta frente al desarrollo de cada individuo.

\section{Desarrollo cognitivo.}

El desarrollo cognitivo siempre ha sido un tema constante de estudio, existen múltiples teorías que tratan de explicar aspectos cruciales como el pensamiento y el lenguaje; en el presente trabajo se abordarán conceptos relacionados con el desarrollo cognitivo, centrando la mira en las definiciones de Lev vygotsky.

En la visión vygotskyana el desarrollo cognitivo se concibe como el proceso por el que el niño va apropiándose de los conocimientos, metas, actividades y recursos culturales de pensamiento y de conducta que la sociedad o comunidad en que vive ha desarrollado para su supervivencia; de manera que es a través de este proceso que implica una "internalización" personal de ese bagaje sociocultural que se le transfiere -como se convierte, de hecho, en un miembro más de la misma (Gutiérrez, 2005, p. 91).

Se debe destacar que desde la teoría de Vygotsky el desarrollo cognitivo es considerado como la adquisición de destrezas conceptuales, actitudinales y procedimentales, estructuradas por el medio cultural y social que garanticen la participación y subsistencia del individuo dentro de la sociedad.

\section{Planteamiento Metodológico}

El presente trabajo es de tipo investigaciónacción debido a que se realiza una intervención social tratando de dar solución a una problemática específica en la comunidad. Pulido, Ballén y Zúñiga (2007) afirman que: Lainvestigaciónacciónconsisteenelanálisis, la concreción de los hechos y conceptualización de los problemas, bases para la planificación de programas de acción, la ejecución de los mismos y la evaluación de los hechos, lo cual servirá para la definición de los problemas y el reinicio del proceso investigativo (p. 39).

Dicho de otra manera, la investigaciónacción es un proceso continuo y repetitivo que arroja resultados para reconocer nuevas necesidades, y así, proponer estrategias de mejora, o como lo dice Vidal y Rivera (2007) "permite vincular el estudio de los problemas en un contexto determinado con programas de acción social, de manera que se logren de forma simultánea conocimientos y cambios sociales" (p.1).

La población tomada en la presente investigación, está compuesta por los núcleos familiares del barrio La Divisa de la 
comuna trece en la ciudad de Medellín y los integrantes de éstos.

\section{Conclusiones}

Mediante las intervenciones realizadas con las familias se lograron mejorar aspectos importantes en las tres dimensiones:

En el desarrollo afectivo, las familias han comprendidoqueel diálogoylacomunicación asertiva son el camino para mejorar las relaciones interpersonales y mantener un ambiente agradable en el entorno familiar, disminuyendo los castigos físicos y buscando otras alternativas de corrección y disciplina con los hijos. Los niños han disminuido el vocabulario soez, y se dirigen con mayor respeto y afecto a los padres y adultos.

En el desarrollo cognitivo, las familias aprendieron sobre sus derechos y sus deberes, las formas de protección y las rutas de apoyo que deben seguir en algún caso de vulnerabilidad. Así mismo han comprendido que los hábitos en el estilo de vida influyen en los procesos de aprendizaje de los niños y se comprometieron a realizar acompañamiento escolar a sus hijos y a motivar la lectura en ellos.

Finalmente, en el desarrollo sociocultural, tanto padres como hijos han comprendido la importancia de la prevención del consumo de sustancias psicoactivas, a pesar del entorno que los rodea y han reconocido que las actitudes emprendedoras les pueden ayudar en la consecución de recursos para mantener a sus familias y contribuir positivamente en el entorno social.

Los 45 niños que participaron en este proceso investigativo junto con sus familias se vieron fortalecidos al lograr estos cambios y aprendizajes, por lo que sienten que sus familias están más consolidadas. Esto los ha motivado a seguir asistiendo a reuniones, por lo que las estudiantes de licenciatura en Preescolar han decido seguir llevando clases y actividades para los niños mientras se continúa con la tercera fase del proyecto que consiste en evaluar el impacto de las intervenciones realizadas con la población.

\section{Análisis de los resultados}

Partiendo de las problemáticas percibidas en las familias de La Divisa, en la fase 1, tanto de los padres como de los niños en los tres aspectos generales, cognitivo, socio cultural y afectivo, se generaron siete (7) micro proyectos enfocados a las dificultades observadas que puedan ayudar en el mejoramiento de las conductas y comportamientos de los miembros de las familias.

Los micro proyectos planteados fueron:

- Maltrato infantil.

- Comunicación.

- Emprendimiento.

- Nutrición

- Derechos y deberes.

- Sustancias psicoactivas.

- Plan lector.

En cada microproyecto se plantearon estrategias y actividades para fortalecer a las familias y capacitarlas para que puedan empoderarse y mejorar su convivencia en la comunidad.

Se lograron realizar 23 intervenciones directas en la población, de las cuales 15 fueron intervenciones con los niños y 8 fueron intervenciones con los padres. El trabajo realizado con los niños desde el plan de acción se desarrolló con base en actividades lúdicas, pedagógicas, didácticas y artísticas. El trabajo realizado con los padres 
a través de los seminarios, charlas y talleres, les permitió reconocer sus necesidades y plantear algunas estrategias para trabajar dentro de cada familia.

Los resultados se analizaron desde cada micro-proyecto:

En cuanto a comunicación familiar, los niños comprendieron los temas de: respeto a los padres y la sana comunicación con ellos, cada niño se comprometió a aplicar lo aprendido en la convivencia dentro del hogar.

En el caso de los padres se tuvo la oportunidad de abordar el tema de la comunicación asertiva, la cual fue de gran impacto y aprendizaje para ellos, esto se pudo evidenciar en la retroalimentación realizada, donde se mostraron que habían aprendido las estrategias comunicativas para tener una sana convivencia familiar.

Los resultados obtenidos en cuanto al maltrato infantil muestran que los niños ahora reconocen sus emociones lo que les permite identificar las formas de maltrato y las rutas de apoyo que pueden seguir.

Con respecto a los padres en cuanto a este tema, de igual forma pudieron aprender más acerca de las rutas de atención en casos de abuso y maltrato infantil. Así mismo pudieron conocer algunas alternativas de corrección y disciplina para sus hijos.

En conocimiento de derechos y deberes se pudo notar que ahora los niños tienen un reconocimiento de los derechos y deberes que poseen como niños y ciudadanos colombianos, conocen a dónde deben acudir cuando estos son vulnerados y qué obligaciones tienen al cumplir los deberes que les son asignados.
Con los padres de familia se pudo observar que la mayoría de ellos no tenían conocimiento de los derechos que les corresponden, por esta razón muchas veces no sabían si estos eran vulneradosy no tenían claro las rutas de apoyo que pueden tomar cuando alguno de estos era vulnerado; este resultado es preocupante porque los padres son la primera fuente de conocimiento para los niños y si ellos no poseen esta información, será más difícil que los niños reconozcan sus derechos y las formas de protección de los mismos. Debido a lo anterior, se enseñaron las rutas de apoyo que pueden seguir cuando sus derechos sean vulnerados, teniendo en cuenta que la familia juega un papel importante al ser el cerco de protección para los niños.

Los niños reconocen la importancia de tener una adecuada higiene oral, hábitos de alimentación saludable y la protección del entorno.

Por medio de la realización de un expo salud los padres de familia aprendieron pautas para mejorar sus estilos de vida. Se pudo observar que los padres se sintieron agradados con la información que se les otorgó, esto mostró que tenían interés en realizar cambios para mejorar sus estilos de vida y el de sus familias. La lectura dentro de la educación es un factor fundamental, la práctica de esta, generará en los niños el deseo de aprender y de obtener más conocimientos, abre la mente y potencializa a los niños a buscar nuevos conocimientos. Los niños aprendieron a crear cuentos, sin importar la temática o la forma de hacerlo (escritos o con dibujos), además de compartir lecturas entre ellos y también con sus familias.

Los padres de familia han comprendido la importancia de realizar acompañamiento en el proceso escolar de sus hijos y se comprometieron a estar más presentes en 
cada una de las actividades que tienen que ver con sus hijos.

Incentivar a los niños a tener un espíritu emprendedor los ayudará a tener metas elevadas y a querer innovar, esto traerá progreso a la comunidad en que ellos estén y al país en general; es por esto que se realizaron con ellos actividades manuales, en las que pudieron aprender diversas técnicas que pueden emplear como oficios.

Estas fueron aceptadas por los niños y realizadas con gran destreza y facilidad, con esta respuesta a la actividad se pudo notar que los niños tienen el deseo de aprender actividades que los motiven a crecer como personas.

El tema de sustancias psicoactivas es sumamente delicado en la población con la que se trabajó, ya que el entorno social y cultural involucra a los jóvenes del sector desde edades tempranas y la población se muestra evitativa ante este tema. Sin embargo, los niños aprendieron sobre el cuidado de sus cuerpos. Los padres conocen la importancia de cuidar a sus hijos mediante alternativas que les ayuden a mejorar su estilo de vida.

\section{Referencias}

Alcaldía de Medellín (2010). Plan de Desarrollo local de la comuna 13. Recuperado de: https://www.medellin. gov.co/MapGIS/OAM/Comuna/Plan/13/CARTILLA_ PDLC13_final.pdf

Belart, A. \& Ferrer, M. (2012). El ciclo de la vida: una visión sistémica de la familia (7a. ed.). España: Desclée de Brouwer.

Bowlby, J. (2014). Vínculos Afectivos: Formación, Desarrollo y Pérdida, 6ta Edición. Madrid: Ediciones Morata, S.L.

Bronfenbrenner, U. (1987). La Ecología del Desarrollo Humano, Experimento en Entornos Naturales y Diseñados. Barcelona, España: PAIDÓS.

García, T. (2013). Uso y Acepciones del Concepto Familia: Entre el Texto y la Realidad. Revista Facultad de Trabajo Social. 29(29). 49-64.

Gutiérrez Martínez, F. (2005). Teorías del desarrollo cognitivo. Barcelona, España: McGraw-Hill.

Maglio, M. \& Graiño, P. (2015). El camino para una lactancia y crianza feliz. Buenos Aires, Argentina: Penguin Random House.

Pulido, R., Ballén, M. \& Zúñiga, F. S. (2007). Abordaje hermenéutico de la investigación cualitativa. Teorías, procesos, técnicas. Bógota, Colombia: Editorial Universidad Cooperativa de Colombia.

Vidal, M. \& Rivera, N. (2007). Investigación-acción. Educación Médica Superior, 21(4) Recuperado de: http://scielo.sld.cu/scielo.php?script=sci_arttext\&pid=S0864-21412007000400012\&lng=es\&tlng=es. 